\title{
Wireless Sensor Network in Application to Air Purifier
}

Gang $W U^{1,2, a}$, Chengdong $W U^{2, b}$, Wenjuan $X U^{3, c}$

\author{
${ }^{1}$ Academy of Information \& Engineering, Shenyang University, Shenyang 110044, China \\ ${ }^{2}$ Academy of Information Science \& Engineering, Northeastern University, Shenyang 110819, \\ China \\ ${ }^{3}$ Communication Department, Shenyang Artillery Academy, shenyang 110867, China \\ a email:hugwg@163.com, b email: wuchengdong@ise.neu.edu.cn, ${ }^{\mathrm{c}}$ email:hugwgaa@tom.com
}

Keywords: wireless sensor network, temperature, monitor, communication, purify

\begin{abstract}
An air purifier is made by temperature, humidity, smoke, carbon dioxide sensors and wireless communication network to the monitoring of the air quality. The obtained parameters compare with standard data to decide whether automatically run the device to generate negative ions or not. Meanwhile, the real-time data are sent to the controller to be saved. The system can generate negative ions to purify the polluted air. The operator gets data by this system. The system is used in factories, banks, airports, railway stations, laboratories, hospitals and other crowded hall for air quality testing and quality improvement. It provides a better air environment for people .Finally, the experiments verify that the system has the stability and robustness.
\end{abstract}

\section{Introduction}

The wireless sensor network (WSN) is a specific use of radio communications systems where many stations nodes transmit the information to a base station (sink) [1]. It requires a completely different approach to radio communications than traditional systems, or even ad hoc networks [2]. You can list a number of important factors affecting the design of the WSN network. These are: bands and communication frequencies, the demand for power supply, reducing external (environmental) as well as, hardware limitations, scalability, fault tolerance range [3]. On the one hand the WSN network is characterized by the architectural and communication specificity associated with the system requirements [4].Basically, the use of WSN in specific applications often requires individual solutions to many complex problems [5][6].

Air quality is one of the most important indicators people concern. In recent years along with the haze aggravated, PM2.5 and continued increases in insoluble particles in the air, increase in the number of cars led to a large number of exhaust emission from inadequate gasoline combustion. The factory does not discharge standards. This causes serious air pollution for people's lives.

With the development of WSN and electronic technology, wireless sensor network is used to monitor air quality, such as in hospital, bank, factory, laboratory, airport lounge, station hall and other public places. A system is studied that can purify the air for environmental monitoring and air quality improvement to protect people's working, living environment. It contributes to improve overall air quality.

This paper is organized as follows. In Section 2, the system model is defined, we give an overview of the adaptive scheme and the project scope is defined. In Section 3, the form of data transmission is mentioned and data analysis is done in section 4 while section 5 deals with a conclusion.

\section{Module Structure}

The system presents an application of WSN networks with random access nodes to monitor the air. It includes two parts, monitor and detecting nodes. Shown in Fig.1.The nodes are distributed in the hall. Each node can detect the temperature, humidity, smoke and carbon dioxide concentration and send the parameter to the monitor. 
When the concentration of smoke, PM2.5, CO2 in the air exceeds the normal standard, the control module in the node controls the relay on, makes the fly back transformer (FBT) producing high-voltage and it provides to negative ions generator make its work. The negative ions are sent to air by fan row to purify air. The detected data by sensor (temperature, humidity, carbon dioxide and dust concentration), and the situation of negative ion generators is transmitted by radio communication to monitor. Monitor displays the parameter from nodes.

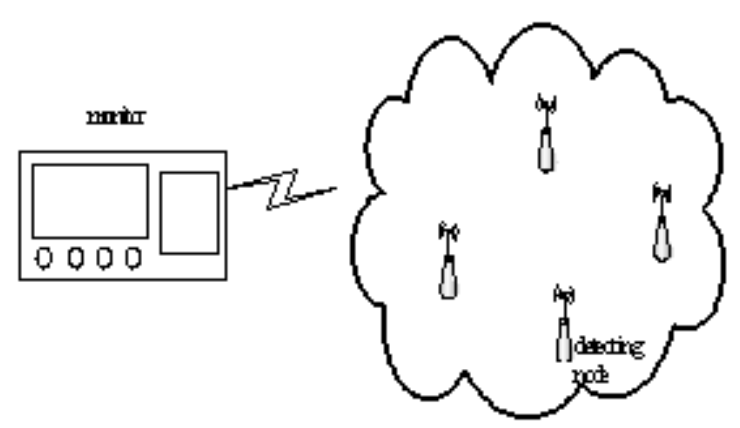

Fig.1.System structure diagram

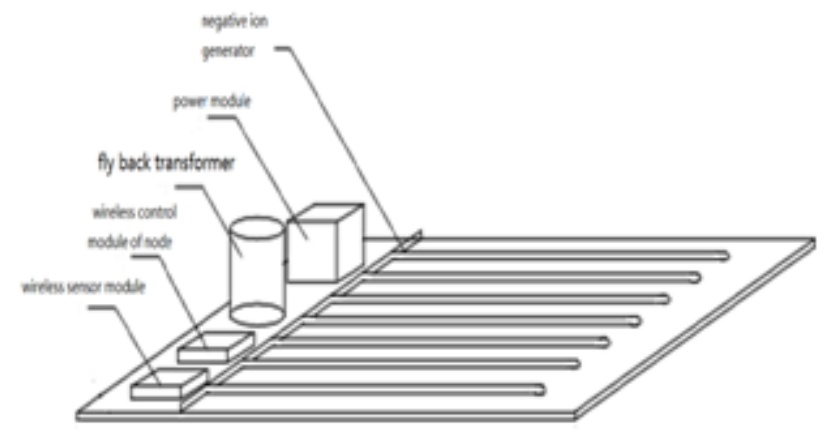

Fig.2.Diagram of bottom panel of node

The bottom panel of the node is equipped with power module, fly back transformer, wireless control module of node, wireless sensor module and negative ions generator. Shown in Fig.2. Display, keys and fans are on the cover, shown in Fig.3.
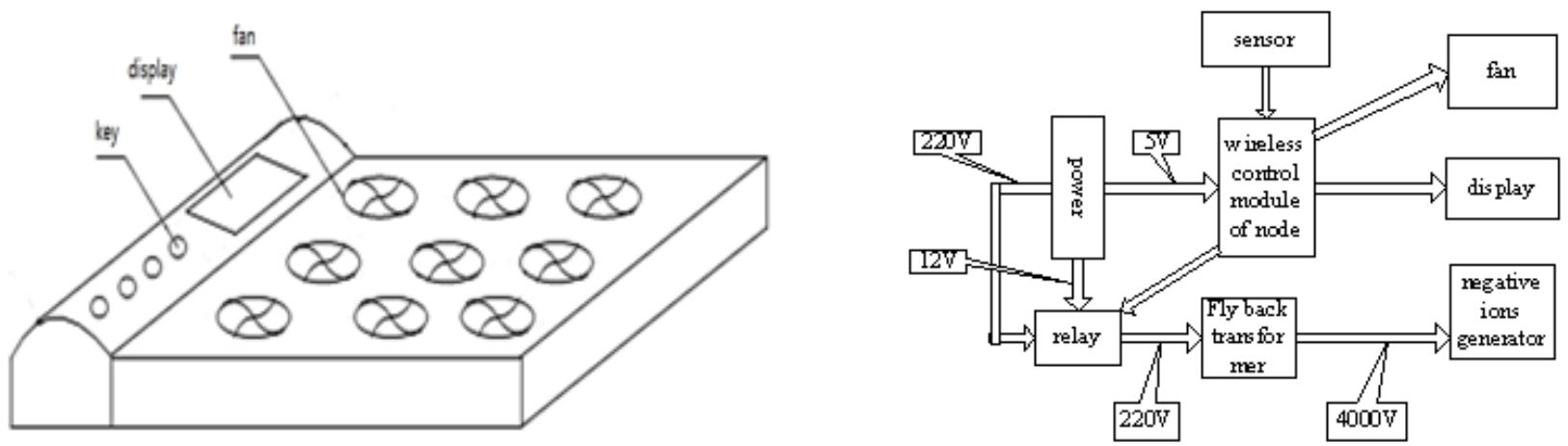

Fig.3.Diagram of top panel of node

Fig.4.Structure diagram of signal flow and control

Power supply module can also provide 220V AC and 12V, 5V DC, provide relay with 12V DC power supply. Relay connects power and FBT. If the relay begins to work, 220V AC reaches the FBT and FBT to generate 4000v for negative ions generator. Sensors, fans and display get 5V DC from power supply module. Wireless control module of node gets the information from sensor and if it reaches the value of work condition, the relay is on. Negative ions generator is working and wireless control module of node gives command to let fan and display on. Shown in Fig.4.

The system contains several wireless modules. Where, wireless modules are composed by control unit, sensor unit, cc2530 micro controller, RF communication unit and power supply unit. The type of wireless module includes temperature, pressure, level, solenoid valve. Number and type of selection can be customized depending on the specific application. Controller CC2530F256 is an on chip system solution with $2.4 \mathrm{GHz}$ RF communications. The wireless module structure is illustrated in Fig.5.

CC2530F256 with RF Transceiver and industry- standard enhanced 8051 CPU has programmable 256KB Flash ROM, 8KB RAM 8 inputs and configurable 12-bit ADC resolution.

\section{Form of Data Transmission}

The radio communication is based on sending short packets of information. From the point of view of the methods of access to the network node, presented probabilistic model protocol should be as short as possible, but it must contain the necessary information. This information should 
contain: the number of the node (sender), which will be associated with particular parameter, the identification number of the test. For each number field test data should be provided with a fixed length. In our case, the data field length is one octet. Taking into account that in the one octet data field is one bit for parity at the disposal of 7 bits, 128 levels determine the value of a given size. This option is the minimum that it can always be expanded at the expense of longer duration of the protocol or at the expense of the occupied bandwidth. It is recommended that a fixed length protocol, which somewhat simplifies operation algorithm and provides a simple mechanism for some kind of validation controls communication with the parity bits. In the proposed model, the duration of the protocol is $t=3.2 \times 10^{-5} \mathrm{~s}$. Excessive expansion of the protocol is not necessary and not advisable because it deteriorates the quality of network communication.

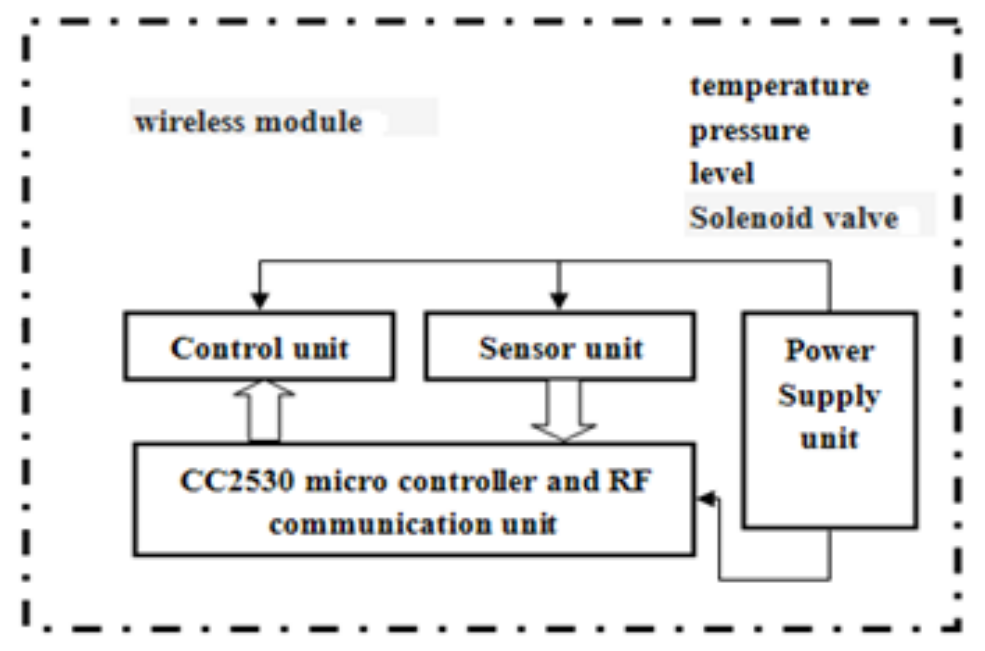

Fig.5.Wireless module structure diagram

\section{Data Base Analysis of Running}

The following Fig. 6 and Fig.7 presents the results of simulation results of a wireless network. In the waiting room of $3000 \mathrm{~m}^{2}, 8$ air purifiers were installed. According to data obtained from sensor in Fig. 6, the solid line shows the concentration of $\mathrm{O} 2$ calculated by used purifiers. The dashed lines are the results of the concentration of $\mathrm{O} 2$ which unused it. It is obviously that the concentration of $\mathrm{O} 2$ was promoted and maintained at a relatively stable level by using the device. In the case of not using the apparatus $\mathrm{O} 2$ levels dropped so that the body feels uncomfortable.

In Fig.7, the solid line shows the concentration of $\mathrm{CO} 2$ calculated by used purifiers. The dashed lines are the results of the concentration of $\mathrm{CO} 2$ which unused it. By suing the device, it improves the content of $\mathrm{CO} 2$ in the air.

All kinds of people stay and wait for train in the waiting room, so it is hard to smell the odor in the air. By using the device, it is significantly reduced odor in the air.

\section{Conclusion and Future Work}

It is presented the model of the wireless sensor network in application to monitoring air in special area. Different from the existing models, this system monitors the state of the air by several nodes with device of negative ions generator. The device working or not is controlled by sensor. The data which are got from sensors are analyzed timely .This system gets good results from actual application. In the using area, the quality of air is better than outside.

In our future work on application WSN networks to purifier we can see two main goals. We will improve the existing models of the wireless sensor networks. The models will be based on accuracy of got parameters of wireless sensor networks. This will let investigate other applications of the WSN in this field.

The second goal will be using wireless sensor network, based on presented models, integrated 
with information system. This will allow using different kinds of computational intelligence methods to optimize performance of the wireless air monitoring systems.

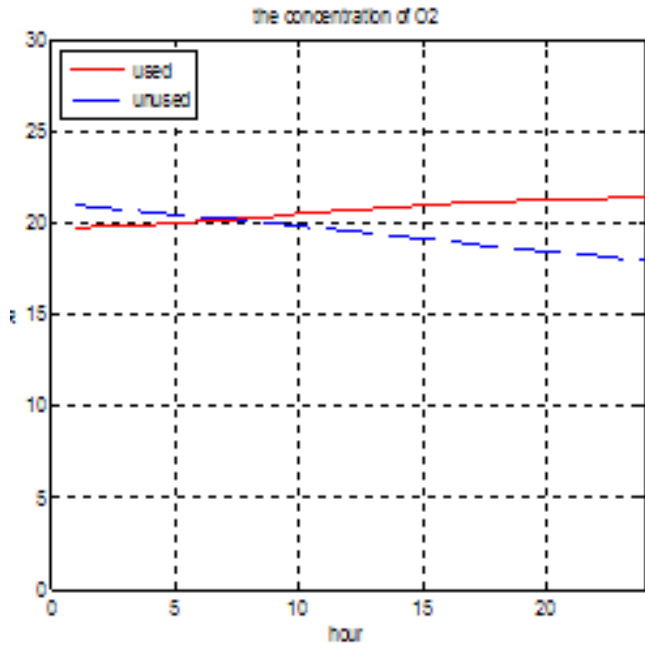

Fig.6.The concentration of $\mathrm{O} 2$

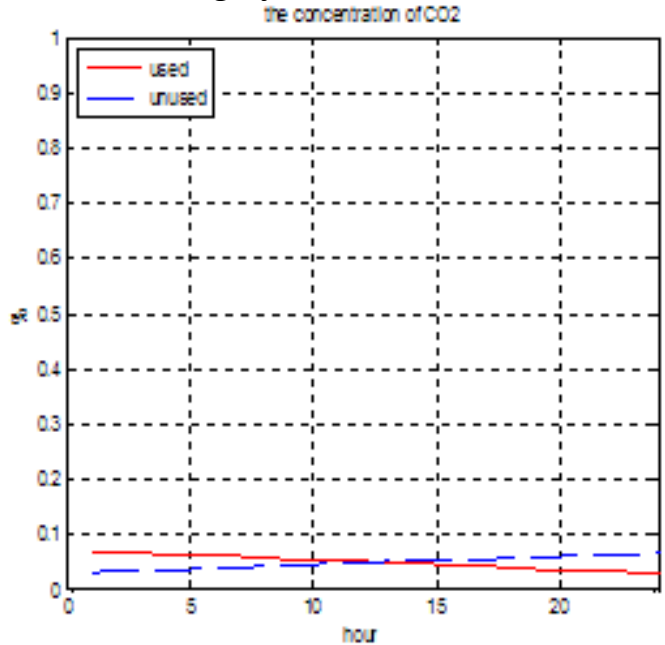

Fig.7.The concentration of $\mathrm{CO} 2$

\section{References}

[1] Akyildiz I. F., Su W., Sankarasubramaniam Y., Cayirci E., Wireless sensor networks: a survey, Computer networks, Vol. 38, No 4, 393-422, 2002

[2] Chatzigiannakis I., Kinalis A., Nikoletseas S.E., Efficient data propagation strategies in wireless sensor networks using a single mobile sink, Computer Communications,Vol. 31, No. 5, 896-914, 2008

[3] Jirong Li, Fujin Li, Yanwei Wu, Zhiwu Yu, The Design of Mine Safety Monitoring System Based on Wireless Sensor Networks, Chinese Journal of Sensor and Actuators, Vol.24, No.9, 1336-1340, 2011

[4] Jang W.-S., Healy W. M., Skibniewski M. J., Wireless sensor networksas part of a web-based building environmental monitoring system,Automation in Construction, Vol. 17, No.6, 729-736, 2008

[5] Misra S., Misra C.S., Woungang I., Algorithmic and theoretical aspects of wireless ad hoc and sensor networks, Computer Communications, Vol.31, No. 4, 655-658,2008

[6] Gupta P., Kumar P.R., The capacity of wireless networks, IEEE Transactions on Information Theory,Vol.46,No.7, 388-404,2000 